\title{
The Characterization for Symmetric Tight Frames and Periodic Gabor Frames and Applications in Computer Engineering \\ Deyou Yuan
}

\author{
College of Math. \& Physics, Nanyang Institute of Technology, Nanyang 473004, China
}

zas123qwe@126.com

Keywords: symmetric frames, symmetric filterbanks, multire solution analysis, wavelet frame subspace, time-frequency analysis, dual Gabor frames, Sobolev space

\begin{abstract}
Materials science is an interdisciplinary field applying the properties of matter to various areas of science and engineering. In this paper, we discuss a new set of symmetric tight frame wave -lets with the associated filterbanks outputs downsampled by several generators. The frames consist of several generators obtained from the lowpass filter using spectral factorization, with lowpass fil -ter via a simple approach using Legendre polynomials. The filters are feasible to be designed and offer smooth scaling functions and frame wavelets. We shall give an example to demonstrste that so-me examples of symmetric tight wavelet frames with three compactly supported real-valued sym-metric generators will be presented to illustrate the results.
\end{abstract}

\section{Introduction}

Manufacturing engineering is an interdisciplinary field applying the properties of matter to var -ious areas of science and engineering. Mechanical engineers apply the principles of mechanics and energy to the design of machines and devices: Energy and Motion. The frame theory has been one of powerful tools for researching into wavelets. Duffin and Schaeffer introduced the notion of frames for a separable Hilbert space in 1952. Later, Daubechies etal revived the study of frames in[1,2], and since then, fra -mes have become the focus of active research, both in theory and in applications, such as signal processing, image processing and sampling theory. The rise of frame theory in applied mathematics is due to the flexibility and redundancy of frames, where robustness, error tolerance and noise suppression play a vital role $[3,4]$. The concept of frame multiresolution analysis (FMRA) as described in [2] generalizes the notion of MRA by allowing non-exact affine frames. However, subspaces at different resolutions in a FMRA are still generated by a frame formed by translates and dilates of a single function. This paper is motivated from the observation that standard methods in sampling theory provide examples of multiresolution structure which are not FMRAs. Inspired by [2] and [5], we introduce the notion of a generalized multiresolution structure(BGMS) of $L^{2}\left(R^{2}\right)$, which has a pyramid decomposition scheme. It also lead to new constructions of affine frames of $L^{2}\left(R^{2}\right)$.

\section{Fundamental Properties of Gabor Frames}

Let $W$ be a separable Hilbert space and $\Lambda$ is an index set. We recall that a sequence $\left\{\lambda_{v}: v \in Z\right\} \subseteq W$ is a frame for $\mathrm{H}$ if there exist positive real numbers $B, C$ such that

$$
\forall \hbar \in W, \quad B\|\hbar\|^{2} \leq \sum_{v \in \Lambda}\left|\left\langle\hbar, \lambda_{v}\right\rangle\right|^{2} \leq C\|\hbar\|^{2}
$$

A sequence $\left\{\lambda_{v}: v \in Z\right\} \subseteq W$ is a Bessel sequence if (only) the upper inequality of (1) holds. If only for all $\hbar \in \Omega \subset W$, the upper inequality of (1) holds, the sequence $\left\{\lambda_{v}\right\} \subseteq W$ is a Bessel sequence with respect to (w.r.t.) $\Omega$. If $\left\{f_{v}\right\}$ is a frame, there exists a dual frame $\left\{f_{v}^{*}\right\}$ such that 


$$
\forall \Upsilon \in W, \quad \Upsilon=\sum_{v \in \Lambda}\left\langle\Upsilon, f_{v}\right\rangle f_{v}^{*}=\sum_{v \in \Lambda}\left\langle\Upsilon, f_{v}^{*}\right\rangle f_{v}
$$

To state our results, the Fourier transform of an integrable function $f(x) \in L^{1}\left(R^{2}\right)$ is defined by

$$
F f(\omega)=\hat{f}(\omega)=\int_{R^{2}} f(x) e^{-2 \pi i x \omega} d x, \quad \omega \in R^{2}
$$

which, as usual, can be naturally extended to functions in $L^{2}\left(R^{2}\right)$. For a sequence $c=\{c(v)\} \in \ell^{2}(Z)$, we define its discrete-time Fourier transform as the function in $L^{2}(0,1)^{2}$ by

$$
F C(\omega)=C(\omega)=\sum_{v \in Z^{2}} c(v) e^{-2 \pi i x \omega} d x
$$

For any $f(t) \in L^{2}(R)$, the novel fractional Fourier transformis defined to be

$$
\begin{gathered}
F_{\alpha}(u)=\mathcal{F}^{\alpha}\{f(t)\}(u)=\int_{\mathbb{R}} f(t) \mathcal{K}_{\alpha}(u, t) d t \\
\psi_{\alpha, m, n}(t)=a_{0}^{-m / 2} \psi\left(a_{0}{ }^{-m} t-n b_{0}\right) e^{-i\left[\left(t^{2}-\left(n a_{0}{ }^{m} b_{0}\right)^{2}\right) / 2\right] \cot \alpha}
\end{gathered}
$$

A system $\left\{g_{m, n}\right\}=\left\{T_{n a} E_{m b} g\right\}$ is a Gabor frame or Weyl-Heisenberg frame for $L^{2}\left(R^{2}\right)$, if there exist two constants $A, B>0$ such that

$$
A\|\hbar\|^{2} \leq \sum_{m, n \in Z^{2}}\left|\left\langle\hbar, g_{m, n}\right\rangle\right|^{2} \leq B\|\hbar\|^{2}
$$

holds for all $\forall \hbar \in L^{2}\left(R^{2}\right)$. For a Gabor frame $\left\{g_{m, n}\right\}$ the analysis mapping (also called Gabor transform) $U_{g}$, given by $U_{g}: f \rightarrow\left\{<f, g_{m, n}>\right\}_{m, n}, \forall f \in L^{2}\left(R^{2}\right)$ and its adjoint, the synthesis mapping (also called Gabor expansion) $U_{g}^{*}$, given by

$$
U_{g}^{*}:\left\{c_{m, n}\right\} \rightarrow \sum_{m, n} c_{m, n} g_{m, n}, \quad \forall\left\{c_{m, n}\right\} \in \ell^{2}\left(Z^{2}\right)
$$

are bounded linear operators. The Gabor frame operator $S_{g}$ is defined by $S_{g}=U_{g}^{*} U_{g}$. Explicitly,

$$
S_{g} f \rightarrow \sum_{m, n}<f, g_{m, n}>g_{m, n}, \quad \forall f \in L^{2}\left(R^{2}\right)
$$

If $\left\{g_{m, n}\right\}$ forms a Gabor frame for $L^{2}\left(R^{2}\right)$, then $\forall f \in L^{2}\left(R^{2}\right)$ can be written as

$$
f=\sum_{m, n}<f, g_{m, n}>h_{m, n}=\sum_{m, n}<f, h_{m, n}>g_{m, n},
$$

where $h_{m, n}$ are the elements of the dual frame, given by $h_{m, n}=S^{-1} g_{m, n}$. Equation (5) provides a constructive answer how to recover $f$ from its Gabor transform $\left\{<f, g_{m, n}>\right\}_{m, n}$ for given analysis window $g$ and how to compute the coefficients in the series expansion $f=\sum_{m, n \in Z} C_{m, n} g_{m, n}$ for given atom $g$. The key is the corresponding dual frame $\left.\left\{<f, g_{m, n}\right\rangle\right\}_{m, n}$. A detailed analysis of Gabor frames brings forward some features that are basic for a further understanding of Gabor analysis. Most of these features are not shared by other frames such as wavelet frames.

\section{Bivariate Generalized Multiresolution Structure}

To characterize such a BGMS, we first introduce the concept of pseudoframes of translates.

Definition 1. Let $\left\{T_{v a} \Upsilon, v \in Z^{2}\right\}$ and $\left\{T_{v a} \widetilde{\Upsilon}, v \in Z^{2}\right\}$ be two sequences in $L^{2}\left(R^{2}\right)$. Let $U$ be a closed subspace of $L^{2}\left(R^{2}\right)$. We say $\left\{T_{v a} \Upsilon, v \in Z^{2}\right\}$ forms an affine pseudoframe for $U$ with respect to $\left\{T_{v a} \tilde{\Upsilon}, v \in Z^{2}\right\}$ if 


$$
\forall f(x) \in U, f(x)=\sum_{v \in Z}\left\langle f, T_{v a} \widetilde{\Upsilon}\right\rangle T_{v a} \Upsilon(x)
$$

Define an operator $K: U \rightarrow \ell^{2}\left(Z^{2}\right)$ by

$$
\forall f(x) \in U, \quad K f=\left\{<f, T_{v a} \Upsilon>\right\},
$$

and define another operator $F: \ell^{2}\left(Z^{2}\right) \rightarrow W$ such that

$$
\forall c=\{c(v)\}_{v \in Z^{2}} \in \ell^{2}\left(Z^{2}\right) . \quad F c=\sum_{v \in Z^{2}} C(v) T_{v a} \tilde{r} .
$$

Theorem 1. Let $\left\{T_{v a} \Upsilon\right\}_{v \in Z^{2}} \subset L^{2}\left(R^{2}\right)$ be a Bessel sequence w.r.t. the subspace $U \subset L^{2}\left(R^{2}\right)$, and $\left\{T_{v a} \widetilde{\Upsilon}\right\}_{v \in Z^{2}}$ is a Bessel sequence in $L^{2}\left(R^{2}\right)$. Assume $K$ be defined by (7), and $F$ be defined by(8). Assume that $P$ is a projection from $L^{2}\left(R^{2}\right)$ onto $U$. Then $\left\{T_{v a} \Upsilon\right\}_{v \in Z^{2}}$ is pseudoframes of translates for $U$ with respect to $\left\{T_{v a} \widetilde{\Upsilon}\right\}_{v \in Z^{2}}$ if and only if

$$
K F P=P \text {. }
$$

Proof. The convergence of all summations of (7) and (8) follows from the assumptions that $\left\{T_{v a} \Upsilon\right\}_{v \in Z^{2}}$ is a Bessel sequence with respect to the subspace $\Omega$, and $\left\{T_{v a} \widetilde{\Upsilon}\right\}_{v \in Z^{2}}$ is a Bessel sequence in $L^{2}(R)$, with which the proof of the theorem is direct forward.

$$
\begin{aligned}
& \sum_{k \in Z^{n}}\left|\left\langle h, \varphi_{v, k}\right\rangle\right|^{2}+\sum_{\sigma=1}^{\tau} \sum_{k \in Z^{n}}\left|\left\langle h, \psi_{v, k}^{\sigma}\right\rangle\right|^{2} \\
& =\frac{1}{(2 \pi)^{n}} \int_{\left[-3^{v-1}, 3^{v-1}\right]^{n}}\left|\sum_{k \in Z^{n}} \hat{h}\left(\omega+2 \pi 3^{v} k\right) \overline{\hat{\varphi}\left(3^{-v}\left(\omega+2 \pi 3^{v} k\right)\right)}\right|^{2} d \omega \\
& +\frac{1}{(2 \pi)^{n}} \sum_{\sigma=1}^{\tau} \int_{\left[-3^{v^{n-1}}, 3^{3^{n-1}}\right]^{n}}\left|\sum_{k \in Z^{n}} \hat{h}\left(\omega+2 \pi 3^{v} k\right) \overline{\hat{\psi}^{\sigma}\left(3^{-v}\left(\omega+2 \pi 3^{v} k\right)\right)}\right|^{2} d \omega \\
& =\frac{1}{(2 \pi)^{n}} \sum_{\sigma=0}^{\tau} \int_{\left[-3^{v-1}, 3^{v-1}\right]^{n}}\left|\sum_{\beta \in Z^{n}} \hat{h}\left(\omega+2 \pi 3^{v} k\right) \overline{s_{\sigma}\left(3^{-v-1} \omega+2 \pi k / 3\right) \hat{\varphi}\left(3^{-v-1} \omega+2 \pi k / 3\right)}\right|^{2} d \omega \\
& =\frac{1}{(2 \pi)^{n}} \sum_{\sigma=0}^{\tau} \int_{\left[-3^{v-1}, 3^{v-1}\right]^{n}}\left\{\mid R_{0}(\omega) \overline{s_{\sigma}\left(3^{-v-1} \omega\right)}+R_{1}(\omega) \overline{S_{\sigma}\left(3^{-v-1} \omega+\eta_{1}\right)}\right. \\
& \left.+R_{2}(\omega) \overline{s_{\sigma}\left(3^{-v-1} \omega+\eta_{2}\right)}+\cdots+\left.R_{3^{n}-1}(\omega) \overline{s_{\sigma}\left(3^{-v-1} \omega+\eta_{3^{n}-1}\right)}\right|^{2}\right\} d \omega \\
& =\frac{1}{(2 \pi)^{n}} \int_{\left[-3^{v-1}, 3^{v-1}\right]^{n}}\left\{\left|R_{0}(\omega)\right|^{2}+\left|R_{1}(\omega)\right|^{2}+\left|R_{2}(\omega)\right|^{2}+\sum_{j=3}^{3^{n}-1}\left|R_{j}(\omega)\right|^{2}\right\} d \omega \\
& =\frac{1}{(2 \pi)^{n}} \int_{\left[-3^{v-1}, 3^{v-1}\right]^{n}}\left|\sum_{k \in Z^{n}} \hat{h}\left(\omega+2 \pi 3^{v+1} k\right) \overline{\hat{\varphi}\left(3^{-v-1} \omega+2 \pi k / 3\right)}\right|^{2} d \omega \\
& +\frac{1}{(2 \pi)^{n}} \int_{\left[-3^{v-1}, 3^{v-1}\right]^{n}}\left|\sum_{k \in Z^{n}} \hat{h}\left(\omega+2 \pi 3^{v+1} k+3^{v+1} \eta_{1}\right) \overline{\hat{\varphi}\left(3^{-v-1} \omega+2 \pi k / 3+\eta_{1}\right)}\right|^{2} d \omega \\
& +\frac{1}{(2 \pi)^{n}} \int_{\left[-3^{v-1}, 3^{v-1}\right]^{n}}\left|\sum_{k \in Z^{n}} \hat{h}\left(\omega+2 \pi 3^{v+1} k+3^{v+1} \eta_{3^{n}-1}\right) \overline{\hat{\varphi}\left(3^{-v-1} \omega+2 \pi k / 3+\eta_{3^{n}-1}\right)}\right|^{2} d \omega \\
& =\frac{1}{(2 \pi)^{n}} \int_{\left[-3^{\eta}, 3^{\left.3^{\nu}\right]^{n}}\right.}\left|\sum_{k \in Z^{n}} \hat{h}\left(\omega+2 \pi 3^{v+1} k\right) \overline{\hat{\varphi}\left(3^{-v-1} \omega+2 \pi k / 3\right)}\right|^{2} d \omega \\
& =\sum_{k \in Z^{n}}\left|\left\langle h, \varphi_{v+1, k}\right\rangle\right|^{2}<+\infty \text {. }
\end{aligned}
$$




$$
W_{f}^{\alpha}(a, b)==\int_{\mathbb{R}} \sqrt{2 \pi} F_{\alpha}(u) \hat{\psi}^{*}(a u \csc \alpha) K_{-\alpha}(u, b) d b
$$

Theorem 2. Let $f(x), \widetilde{f}(x), \Upsilon_{t}(x)$ and $\widetilde{\Upsilon}_{t}(x), t \in I$ be functions in $L^{2}\left(R^{2}\right)$. Assume that conditions in Theorem 2 are satisfied. Then, for any funct- ion $\Gamma(x) \in L^{2}\left(R^{2}\right)$, and any integer $\mathrm{n}$,

$$
\sum_{k \in Z^{2}}\left\langle\Gamma, \widetilde{f}_{n, k}\right\rangle f_{n, k}(x)=\sum_{i=1}^{80} \sum_{v=-\infty}^{n-1} \sum_{k \in Z^{2}}\left\langle\Gamma, \widetilde{\Upsilon}_{t: v, k}\right\rangle \Upsilon_{t: v, k}(x) \text {. }
$$

Furthermore, for any bivariate function $\Gamma(x) \in L^{2}\left(R^{2}\right), \quad \Gamma(x)=\sum_{i=1}^{80} \sum_{v=-\infty}^{+\infty} \sum_{k \in Z^{2}}\left\langle\Gamma, \widetilde{\Upsilon}_{l: v, k}\right\rangle \Upsilon_{t: v, k}(x)$. Consequently, if $\left\{\Upsilon_{t: v, k}(x)\right\}$ and $\left\{\widetilde{\Upsilon}_{t: v, k}(x)\right\}, \quad\left(l \in \Lambda, v \in Z, \quad k \in Z^{2}\right)$ are also Bessel sequences, they are a pair of affine frames for $L^{2}\left(R^{2}\right)$.

\section{Conclusion}

We characterize the pseudoframes of translates for the subspaces of $L^{2}\left(R^{2}\right)$. The pyramid decomposition scheme is derived based on such a BGMS. As a major new constribution the construction of affine frames for $L^{2}\left(R^{2}\right)$ based on a BGMS is presented.

\section{References}

[1] I. Daubechies, A. Grossmann, A. Meyer, Painless nonorthogonal expansions. J. Math. Phys. 1986;27: 1271-1283.

[2] J. J. Benedetto, S. Li, The theory of multiresolution analysis frames and applications to filter banks. Appl. comput. Harmon.Anal. 1998;5: 389-427.

[3] A.Ron, Z. Shen, Affine systems in L2(Rd). (II) Dual systems. J. Fourier Anal. Appl. 1997, 4: 617-637.

[4] Qingjiang Chen, Zhi Shi, Huaixin Cao. The characterization of a class of subspace pseudo frames with arbitrary real number translations. Chaos, Solitons \& Fractals. 2009, 42(5): 2696-2706.

[5] S. Li, et al, Pseudoframes for Subspaces with Applications. Four Anal Appl 2004;10: 409-431.

[6] S. Li, A Theory of Geeneralized Multiresolution Structure and Pseudoframes of Translates. J. Fourier Anal. Appl. 2001;6(1): 23-40. 